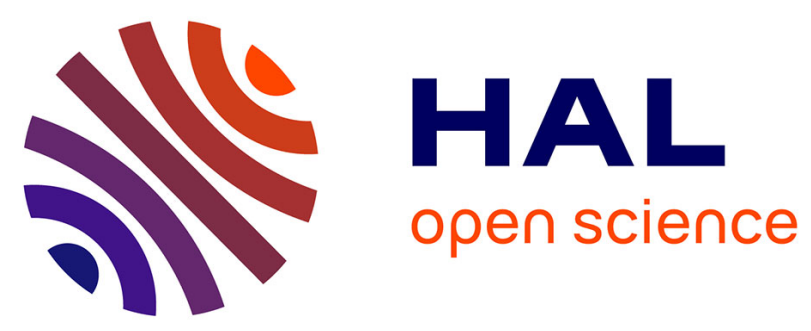

\title{
Thermal boundary layer near roughnesses in turbulent Rayleigh-Bénard convection: flow structure and multistability
}

\author{
Julien Salort, Olivier Liot, Eleonore Rusaouen, Fanny Seychelles, \\ Jean-Christophe Tisserand, Mathieu Creyssels, Bernard Castaing, Francesca \\ Chillà
}

\section{To cite this version:}

Julien Salort, Olivier Liot, Eleonore Rusaouen, Fanny Seychelles, Jean-Christophe Tisserand, et al.. Thermal boundary layer near roughnesses in turbulent Rayleigh-Bénard convection: flow structure and multistability. Physics of Fluids, 2014, 26, pp.015112. 10.1063/1.4862487 . hal-00920342

\section{HAL Id: hal-00920342 \\ https://hal.science/hal-00920342}

Submitted on 18 Dec 2013

HAL is a multi-disciplinary open access archive for the deposit and dissemination of scientific research documents, whether they are published or not. The documents may come from teaching and research institutions in France or abroad, or from public or private research centers.
L'archive ouverte pluridisciplinaire HAL, est destinée au dépôt et à la diffusion de documents scientifiques de niveau recherche, publiés ou non, émanant des établissements d'enseignement et de recherche français ou étrangers, des laboratoires publics ou privés. 


\title{
Thermal boundary layer near roughnesses in turbulent Rayleigh-Bénard convection: flow structure and multistability
}

\author{
J. Salort, ${ }^{1}$ O. Liot, ${ }^{1}$ E. Rusaouen, ${ }^{1}$ F. Seychelles, ${ }^{1,}$ a) $J .-C$. Tisserand, ${ }^{1}$ M. Creyssels,${ }^{1,2}$ B. Castaing,,${ }^{1}$ and F. \\ Chillà ${ }^{1}$ \\ 1) Laboratoire de physique, UMR CNRS 5672, École Normale Supérieure de Lyon, 46 allée d'Italie, \\ 69364 Lyon cedex 7, France, EU \\ ${ }^{2)}$ LMFA, UMR CNRS 5509, École Centrale de Lyon, Université Claude Bernard Lyon I, INSA Lyon, \\ 69134 Écully Cedex, France, EU
}

(Dated: 16 November 2013)

We present global heat-transfer and local temperature measurements, in an asymmetric parallelepiped Rayleigh-Bénard cell, in which controlled square-studs roughnesses have been added. A global heat transfer enhancement arises when the thickness of the boundary layer matches the height of the roughnesses. The enhanced regime exhibits an increase of the heat transfer scaling. Local temperature measurements have been carried out in the range of parameters where the enhancement of the global heat transfer is observed. They show that the boundary layer at the top of the square-stub roughness is thinner than the boundary layer of a smooth plate, which accounts for most of the heat-transfer enhancement. We also report multistability at long time scales between two enhanced heat-transfer regimes. The flow structure of both regimes is imaged with background-oriented synthetic Schlieren and reveals intermittent bursts of coherent plumes.

PACS numbers: 47.27.te, 47.27.nb

Keywords: Bénard convection, Boundary layer turbulence, Confined flow, Natural convection

\section{INTRODUCTION}

Thermal convection is a process that occurs naturally in a wide range of natural systems (convection inside stars, atmospheric motions, circulation in the ocean) and this process also plays an important role in the industry (heat exchangers, indoor air circulation). In these situations, the thermal forcing is very strong and the flow highly turbulent. One possible model system, widely used to study this problem is the Rayleigh-Bénard cell: a fluid layer confined in a closed cell with adiabatic walls, heated from below and cooled from above. Although this is one of the first non-linear model system that was investigated nearly a century ago $^{1}$, the emergence and the role of turbulence inside the boundary layers and their consequences on the global heat transfer are still open for debate ${ }^{2}$.

It is now clear however that the plates and the boundary conditions play a crucial role in the dynamics of the system $^{3-8}$. To understand how instabilities develop near the plates and how they affect the general dynamics of heat transport, one approach is to purposely change the plate properties to trigger transitions in the boundary layers. One possibility is to introduce controlled plate roughnesses. There has been a lot of efforts to figure out how the dynamics of the flow is modified in this situation $^{9-16}$. It is now well established that controlled roughnesses on the plates enhance the global heat transfer and, in some cases, also increase the turbulent convection scaling exponent. This is also of utmost importance,

a) Present address: CORIA, Avenue de l'Université, BP 12, 76801 Saint Étienne du Rouvray, France, EU and thus widely used and studied, in the engineering community as a heat exchange enhancement technique ${ }^{17}$.

In this paper, we investigate how the thermal boundary layers and the flow structure near the plates are modified by the roughnesses. We use a rectangular RayleighBénard cell with a rough bottom plate and a smooth top plate. This allows in-situ comparison of the flow structure with or without the roughnesses. We report both local temperature measurements carried out using miniature thermistors and fluctuations at a larger scale obtained with background-oriented synthetic Schlieren techniques.

\section{EXPERIMENTAL SETUP AND HEAT TRANSFER MEASUREMENTS}

Our convection cell is a $10 \mathrm{~cm}$-thick $40 \mathrm{~cm} \times 40 \mathrm{~cm}$ rectangular cell with $2.5 \mathrm{~cm}$-thick PMMA walls (see sketch in figure 1). Both plates consist in $4 \mathrm{~cm}$-thick copper plates coated with a thin layer of nickel. The bottom plate is Joule-heated, with possible powers up to $610 \mathrm{~W}$. The top plate is cooled with a temperature regulated water circulation. The top plate is smooth. The controlled roughnesses on the bottom plate consist in an array of $2 \mathrm{~mm}$-heigh $5 \mathrm{~mm} \times 5 \mathrm{~mm}$ square plots, machined in the copper, evenly spaced every $1 \mathrm{~cm}$. The working fluid is deionized water. The mean temperature can be varied between $15^{\circ} \mathrm{C}$ and $45^{\circ} \mathrm{C}$ (the corresponding Prandtl numbers, $\operatorname{Pr}=\nu / \kappa$, are respectively 8.1 and 3.9).

The control parameter is the dimensionless temperature difference between the hot $\left(T_{h}\right)$ and the cold $\left(T_{c}\right)$ 


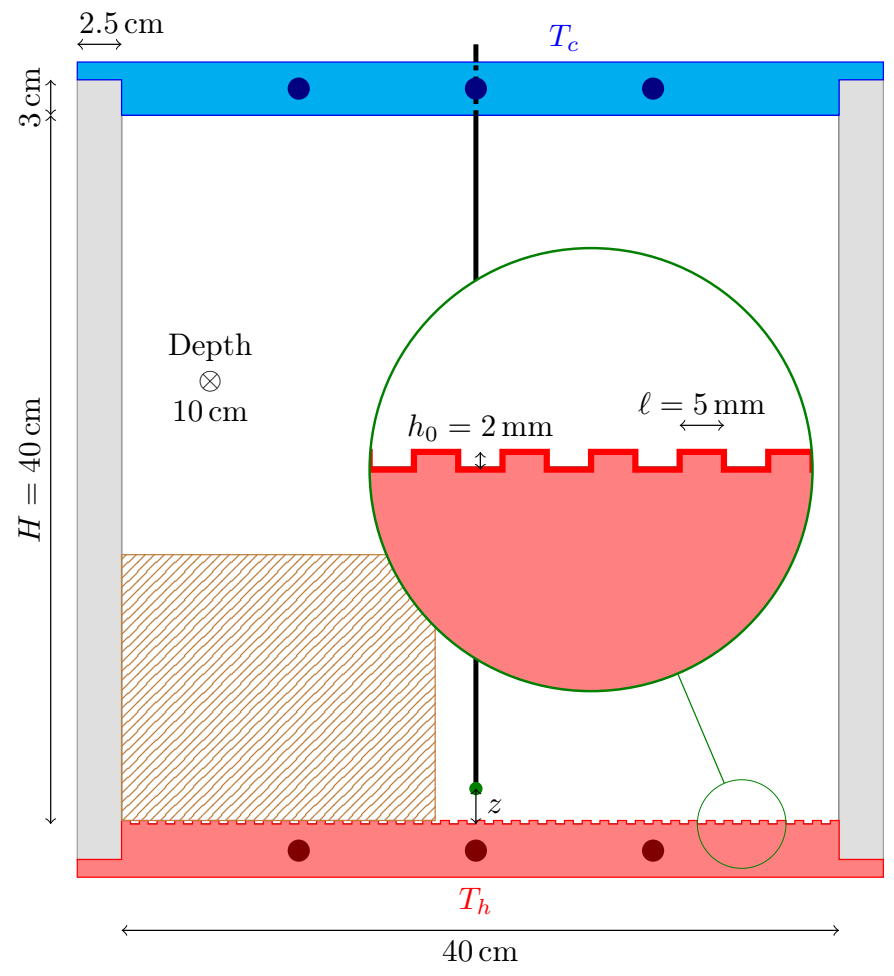

FIG. 1. Sketch of the convection cell. The six dark dots in the plates show the location of the PT100 temperature sensors. The miniature thermistor is symbolized by the dark green circle at a distance $z$ from the bottom plate. The dashed brown region indicates the area when the Schlieren is recorded. The light gray rectangles represent the PMMA walls.

plates, given by the Rayleigh number,

$$
R a=\frac{g \alpha\left(T_{h}-T_{c}\right) H^{3}}{\nu \kappa}
$$

where $H$ is the height of the cell, $g$ is the gravitational acceleration, $\alpha$ the constant pressure thermal expansion coefficient. In the experiment, the heat flux is imposed with the electrical power on the bottom plate. We then measure the temperature drop, $T_{h}-T_{c}$, as well as the temperature of the bulk, $T_{\text {bulk }}$.

The dimensionless global heat transfer response is given by the Nusselt number,

$$
N u=\frac{Q H}{\lambda\left(T_{h}-T_{c}\right)}
$$

which compares the heat flux $Q$ to the purely diffusive flux $\lambda\left(T_{h}-T_{c}\right) / H$, where $\lambda$ is the fluid thermal conductivity. In our case, the typical Rayleigh numbers are in the range $10^{9}-10^{11}$, and the typical Nusselt numbers between 100 and 400 .

Since the cell is not symmetric, given the bulk temperature $T_{\text {bulk }}$, it is possible to define Rayleigh and Nusselt numbers based on the temperature difference between each plate and the bulk as done in the work of Tisserand, et al. ${ }^{15}$. The Rayleigh number associated with the smooth plate is,

$$
R a_{s}=\frac{2 g \alpha\left(T_{\mathrm{bulk}}-T_{c}\right) H^{3}}{\nu \kappa}
$$

and its Nusselt number,

$$
N u_{s}=\frac{Q H}{2 \lambda\left(T_{\text {bulk }}-T_{c}\right)}
$$

And similarly for the rough plate, the Rayleigh number is,

$$
R a_{r}=\frac{2 g \alpha\left(T_{h}-T_{\mathrm{bulk}}\right) H^{3}}{\nu \kappa}
$$

and its Nusselt number,

$$
N u_{r}=\frac{Q H}{2 \lambda\left(T_{h}-T_{\mathrm{bulk}}\right)}
$$

To prevent spurious heat losses, the cell was insulated with several layers of Mylar and placed inside a temperature regulated copper thermal screen. Each plate temperature is obtained with 4-wire measurements of precisely calibrated PT100 resistors located inside the copper plate (see figure 1). The bulk temperature is obtained with a 4-wire measurement of one PT100 resistor placed at the cell mid-height. It is found to be larger than $\left(T_{h}+T_{c}\right) / 2$. The resulting $R a_{s}, N u_{s}, R a_{r}, N u_{r}$ are given in figure 2 . These results are in quantitative agreement with the measurements of Tisserand, et al. carried out in a larger cylindrical cell ${ }^{15}$. A transition toward a regime of enhanced heat transfer is observed on the rough plate for $N u_{r} \approx 100$, corresponding to a boundary layer thickness,

$$
\delta_{t h}=\frac{H}{2 N u} \approx 2 \mathrm{~mm}
$$

which matches the height of the roughnesses.

In the next sections, we investigate the high-Ra regime where the heat transfer enhancement is observed. These are obtained at high power, where the heat losses are small compared to the heat flux through the cell, even if the Mylar insulating layers are removed, which is required to record images with a camera.

\section{BOUNDARY LAYER PROPERTIES}

\section{A. Experimental observations}

The boundary layer structure is investigated with two miniature glass-encapsulated Betatherm GR2KM3187J15 thermistors, $400 \mu \mathrm{m}$ in diameter. They are placed at the end of rigid rods and inserted inside the cell from above. The position can be chosen near the smooth top plate or near the rough bottom plate, either above a plot, in a groove or in a notch where the fluid 


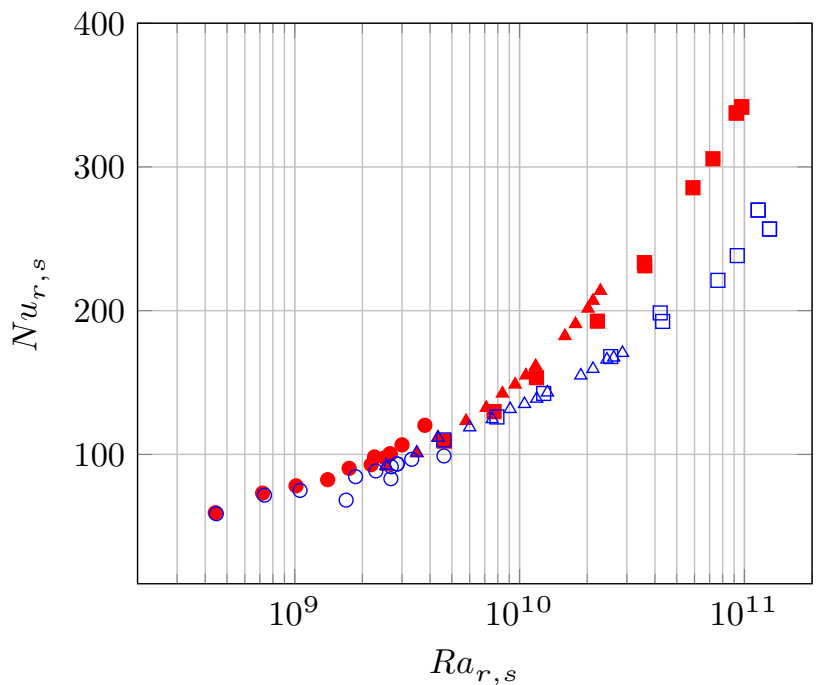

FIG. 2. Dimensionless heat transfer. Circles: $T=15^{\circ} \mathrm{C}$ $(P r=8)$. Triangles: $T=30^{\circ} \mathrm{C}(P r=5.4)$. Squares: $T=$ $45^{\circ} \mathrm{C}(\operatorname{Pr}=3.9)$. Open symbols: $N u_{s}$ versus $R a_{s}$. Filled symbols: $N u_{r}$ versus $R a_{r}$. See text for the notations.

is at rest (see figure 3). The results presented in this section have been obtained with a mean temperature of $40^{\circ} \mathrm{C}$ and a heat power of $400 \mathrm{~W}$. The resulting global temperature difference $T_{h}-T_{c} \approx 20^{\circ} \mathrm{C}$, which corresponds to a global Rayleigh number $R a \approx 5 \times 10^{10}$, and a Nusselt number $N u \approx 250$.

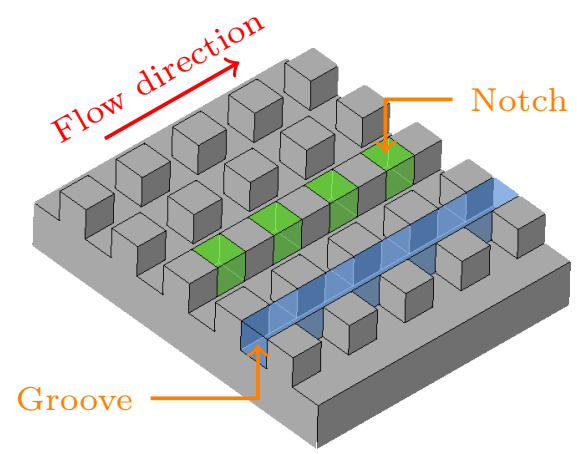

FIG. 3. Schematic view of the rough plate. The blue-shaded part is a groove, washed by the large scale circulation (given by the red arrow). The green-shaded part are notches, where the fluid at rest is not washed by the flow.

The profiles of temperature rms are obtained by moving the thermistor at a distance $z$ away from the plate. The definition of $z$ above a plot, or inside a notch, is schematized in figure 4 . Figure 5 shows the profiles obtained near the smooth plate and near the rough plate.

The profile obtained near the smooth plate shows a peak close to the plate. The absissa of this peak can be
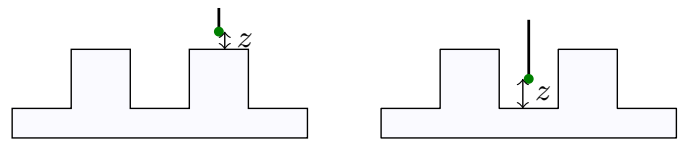

FIG. 4. Definition of $z$ near the rough bottom plate, above a plot, or inside a notch.

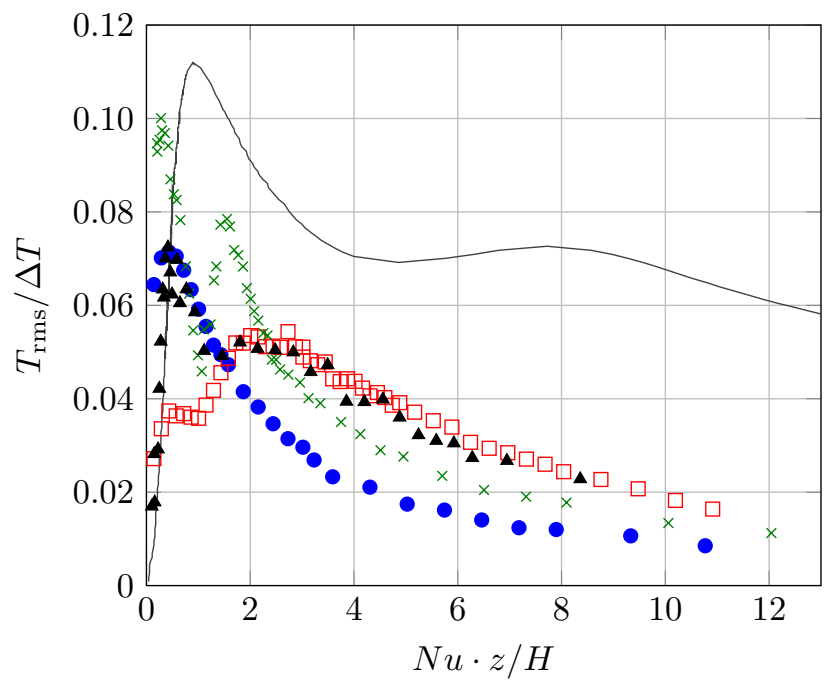

FIG. 5. Profiles of the rms temperature fluctuations near the smooth plate (full circles) and near the rough plate, with the $z$ origin at the bottom of a notch (open squares). The profiles are compared with results from Ciliberto, et al. ${ }^{11}$ (x crosses) and with those of Du, et al. ${ }^{10}$ (black triangles). For reference, the numerical simulation from Stringano, et al. ${ }^{18}$ is shown as solid black line.

used as an estimate of the thermal boundary layer thickness, $\delta_{t h}$. We find $\delta_{t h} \approx 0.8 \mathrm{~mm}$, in fair agreement with the classical estimate (Eq. 7) for this set of parameters ( $H=40 \mathrm{~cm}$ and $N u \approx 250$ ). The rough-case profile is obtained near the rough plate, with the $z$ origin at the bottom of a notch, as in the right sketch in figure 4 . It shows two peaks. This is similar to the profile obtained by $\mathrm{Du}$ and Tong ${ }^{10}$ in a cell with rough upper and lower surfaces, and also in good agreement with the measurement of Ciliberto, et al. ${ }^{11}$, in an asymmetric cell with a rough bottom plate. It is very likely that the slight differences between the profiles come from the details of the roughness features - evenly spaced metallic parallelepipedic roughnesses in our case, versus spheres with random positions and sizes in the work of Ciliberto, et al. or evenly spaced pyramids in the work of $\mathrm{Du}$, et al..

The histograms obtained near the smooth plate are shown in figure 6 . They are similar to those typically obtained with symmetric smooth plates ${ }^{19-24}$. The shape of the histogram is nearly gaussian deep inside the boundary layer. At the edge of the boundary layer $(z=0.8 \mathrm{~mm}$ in our case), the histogram is skewed and does not show 


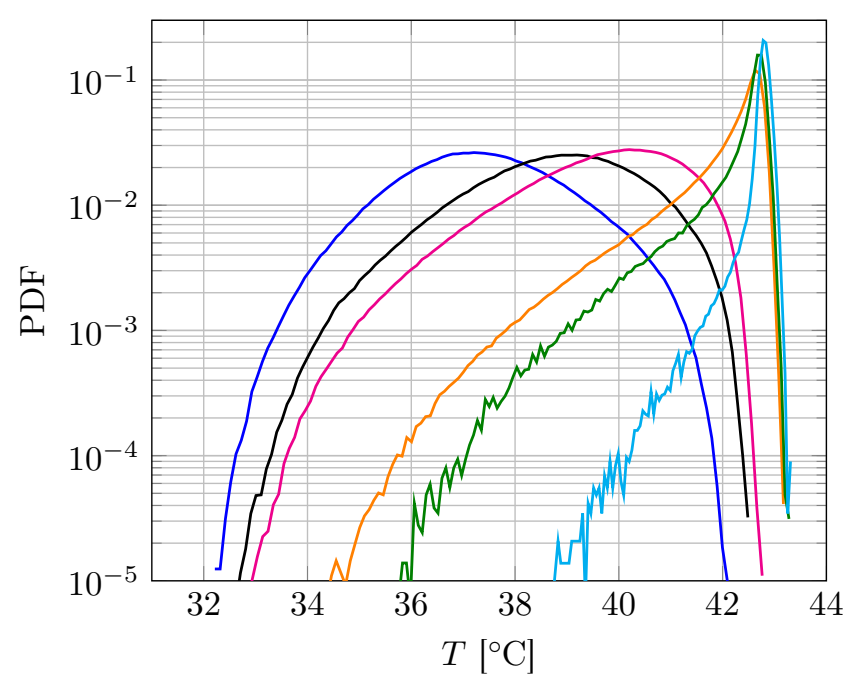

FIG. 6. Histogram of temperature for various distance $z$ away from the top smooth plate. From left to right: $z=0.4 \mathrm{~mm}$, $0.6 \mathrm{~mm}, 0.8 \mathrm{~mm}, 2.0 \mathrm{~mm}, 3.0 \mathrm{~mm}, 10 \mathrm{~mm}$.

an inflection. Further away but still close to the thermal boundary layer $(z>0.8 \mathrm{~mm})$, the histogram is skewed and shows an inflection.

The strong quantitative and qualitative similarities between our measurements near the smooth plate in this asymmetric cell with those obtained in symmetric smooth cells back up the hypothesis of independance of the plates that was used in the analysis of Tisserand, et al. for such an asymmetric Rayleigh-Bénard system ${ }^{15}$.

An order of magnitude of the thermal boundary layer thicknesses can be obtained by extrapolating the qualitative features of the smooth-case temperature histogram to the histograms obtained near the rough plate, shown in figure 7. Du and Tong have shown that the shape of the temperature histograms in rough cells are similar to those of smooth cells, at least in the center of the cell $^{12}$. Above a plot, at the closest possible distance from the plate, the histogram still shows an inflection. This provides an upper bound for the thermal boundary layer thickness above a plot, $\delta_{t h \text {,plot }}$. It is apparently very thin, thinner that can be resolved by our probe,

$$
\delta_{t h, \text { plot }} \lesssim 0.4 \mathrm{~mm}
$$

Inside a notch, on the other hand, the fluctuations remain symmetric until $z=1.4 \mathrm{~mm}$. This indicates that the fluid is indeed rather still inside a notch.

At this stage, the enhancement mechanism that those results suggest is therefore different from the one which was proposed in the work of Tisserand, et al. ${ }^{15}$. The fluid that remains still in the notch constitutes a thermal resistance and therefore probably does not contribute much to the global heat transfer. On the other hand, the thermal boundary layer on the plot is much thinner than the smooth case, which suggests a higher local Nusselt number on these locations.
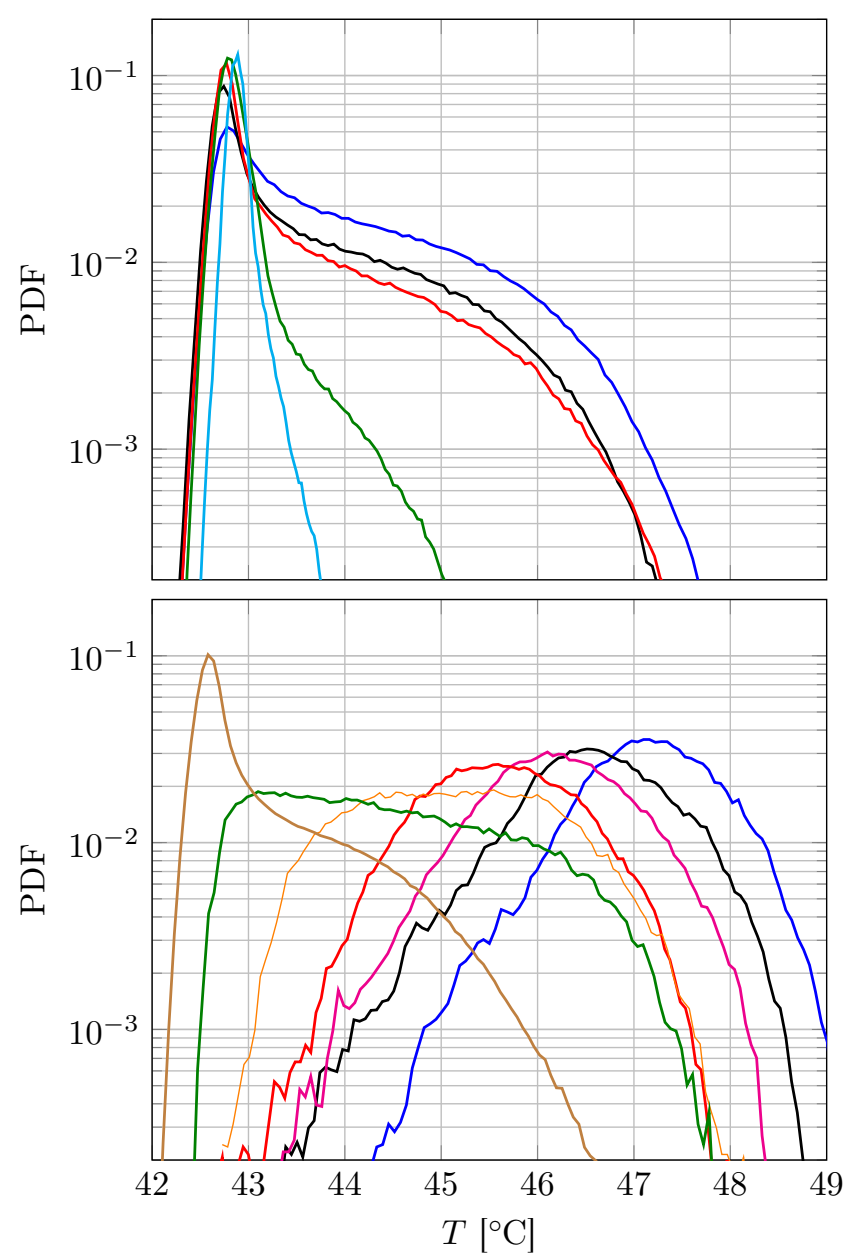

FIG. 7. Histogram of temperature for various distance $z$ away from the rough bottom plate. Upper subplot: above a plot, from right to left $z=0.4 \mathrm{~mm}, 0.6 \mathrm{~mm}, 1.0 \mathrm{~mm}$, $3.0 \mathrm{~mm}, 10 \mathrm{~mm}$. Lower subplot: inside a notch, from right to left, $z=0.4 \mathrm{~mm}, 0.6 \mathrm{~mm}, 0.8 \mathrm{~mm}, 1.4 \mathrm{~mm}, 2.0 \mathrm{~mm}, 3.0 \mathrm{~mm}$, $7.2 \mathrm{~mm}$. See figure 4 for the definition of $z$.

\section{B. Laminar interpretation}

One possible explanation is that the top of the plots behaves as if the corresponding portions of the original smooth plate had been moved higher, inside the bulk. The boundary layer does not develop continuously on the whole plate, but rather on each individual plot. One solution could be a laminar velocity boundary layer developing on the plots, as sketched in figure 8 .

In this situation, the velocity boundary layer, $\delta_{v}$ can be computed from the Prandtl-Blasius equations ${ }^{25}$,

$$
\delta_{v}(x) \approx 3.0 \sqrt{\frac{x \nu}{U}}
$$

The thermal boundary layer lies inside the velocity boundary layer because $\operatorname{Pr}>1$. Thus, the thermal boundary layer thickness relates to the velocity boundary 


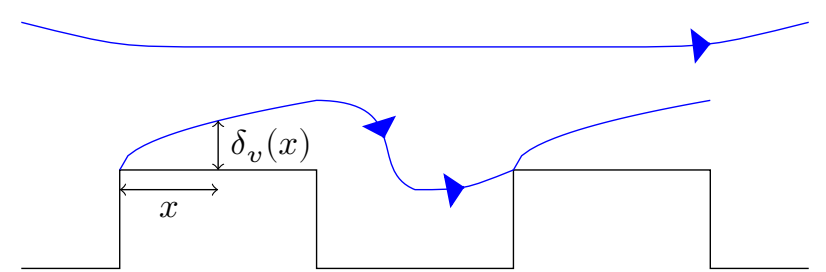

FIG. 8. Sketch of a laminar velocity boundary layer on the top of the plots. Its thickness $\delta_{v}(x)$ can be estimated from Prandtl-Blasius equations (Eq. 9).

layer thickness $\operatorname{as}^{26}$

$$
\delta_{t h}=\delta_{v} \operatorname{Pr}^{-1 / 3}
$$

We can then compute an estimate for the thermal boundary layer thickness on the plot

$$
\delta_{t h, \text { plot }} \approx 3.0 \sqrt{\ell H} \operatorname{Re}^{-1 / 2} \operatorname{Pr}^{-1 / 3}
$$

The Reynolds number can be computed experimentally from velocity measurements obtained both with an instrumented "smart" particle ${ }^{27}$ and with PIV. The full discussion of the velocity data is outside the scope of this paper and will be discussed in a future paper.

The measured velocity in this range of parameter is $U \approx 2 \mathrm{~cm} / \mathrm{s}$, which gives $\delta_{t h \text {,plot }} \approx 0.7 \mathrm{~mm}$ using Eq. 11 , which is larger than the experimental estimate. In addition, there is no experimental evidence that the thermal boundary layer on the plot is larger at the downstream edge of the plot. This means that the laminar velocity boundary layer is not stable. Possible reasons are the divergent streamlines downstream the plot and the sharp edge of the plots. Both are known to cause destabilisation of laminar boundary layers.

\section{Turbulent model}

Since the laminar model appears to fail, we consider another possible interpretation for our experimental observations: the boundary layers may have become turbulent. The shear Reynolds number is a criterion to judge about the potential transition of a boundary layer from laminar toward the turbulent state. It is given by

$$
R e_{s}=\frac{\delta_{v} U}{\nu}
$$

where $\delta_{v}$ is the velocity boundary layer thickness. Direct measurements of this shear Reynolds number have recently been carried out in a smooth cylindrical cell of aspect ratio 1 , at Rayleigh numbers similar to ours by the group of Ilmenau ${ }^{28}$. In particular, at $R a=1.42 \times 10^{10}$, they have measured $R e_{s}=79$ and concluded that this value lies in the range where the boundary layer is laminar.
The Reynolds number may be different in a rectangular cell like ours, but the order of magnitude should stand. In this range of shear Reynolds numbers however, various experiments have shown that roughnesses can lower the critical Reynolds $R e_{S}$ above which the BL becomes turbulent ${ }^{29}$. It is therefore possible to consider that the boundary layer becomes turbulent in our situation. Therefore, we can follow the approach proposed by Kraichnan $^{30}$. The boundary layer is characterised by a critical Péclet number $P e_{T}$ and a critical shear Reynolds number $R e_{S}$. The Péclet number is defined by,

$$
P e_{T}=\frac{U\left(\delta_{t h}\right) \delta_{t h}}{\kappa}
$$

In the range of Prandtl numbers that we can explore, the thermal boundary layer lies within the velocity boundary layer, hence,

$$
P e_{T}=\frac{U \delta_{t h}^{2}}{\kappa \delta_{v}}
$$

The Nusselt number, defined as $N u=H /\left(2 \delta_{t h}\right)$ is thus

$$
N u=\frac{1}{2} \operatorname{RePr}^{1 / 2}\left(P e_{T} R e_{S}\right)^{-1 / 2}
$$

In the case of Kraichnan theory, the laminar boundary layer is destabilised by the turbulence in the bulk. In our case, the destabilisation arises from geometrical reasons. Therefore, we do not expect the critical $P e_{T}$ and $R e_{S}$ to have the universal values they have in the turbulent case, but rather to have geometry-dependent values. Our experimental results, as well as many previous observations in the literature, show that the transition occurs when the thermal boundary layer thickness is $h_{0}$, hence the critical values of the Péclet and Nusselt numbers:

$$
P e_{T}=\frac{h_{0}^{2} U_{c}}{\kappa \delta_{c}}
$$

$$
N u_{c}=\frac{H}{2 h_{0}}
$$

And by definition,

$$
R e_{S}=\frac{\delta_{c} U_{c}}{\nu}
$$

where $U_{c}$ is the mean flow velocity at the transition and $\delta_{c}$ the velocity boundary layer thickness at the transition. From Eq. 16 and 18, we get

$$
P e_{T} R e_{S}=\left(\frac{h_{0}}{H}\right)^{2} \operatorname{Pr} R e_{c}^{2}
$$

Combining Eqs 15 and 19, we obtain

$$
N u=\frac{H}{2 h_{0}} \frac{R e}{R e_{c}}
$$


with $R e=H U_{c} / \nu \propto R a_{c}^{1 / 2} \operatorname{Pr}^{-3 / 4}$, hence

$$
N u=\frac{H}{2 h_{0}} \frac{R a^{1 / 2}}{R a_{c}^{1 / 2}}
$$

Before the transition, the Nusselt versus Rayleigh behavior is similar to a smooth plate. In this range of $R a$ and $P r$, it is close to $N u=\sigma R a^{1 / 3}$, thus

$$
R a_{c}=\left(\frac{N u_{c}}{\sigma}\right)^{3}
$$

This allows Eq. 21 to be rewritten:

$$
N u=\frac{(2 \sigma)^{3 / 2}}{2}\left(\frac{h_{0}}{H}\right)^{1 / 2} R a^{1 / 2}
$$

In the following, we will use reduced variables: reduced Rayleigh number $r$ and reduced Nusselt number $n$, defined as,

$$
\begin{aligned}
r & =\left(\frac{h_{0}}{H}\right)^{3} R a \\
n & =\left(\frac{H}{2 h_{0}}\right) N u
\end{aligned}
$$

In these reduced variables, Eq. 23 can be rewritten without explicit use of the geometrical parameters of the roughnesses :

$$
n=(2 \sigma)^{3 / 2} r^{1 / 2}
$$

To determine $\sigma$, we can use the data obtained by Roche, et al. in a cell with V-shape groove roughnesses ${ }^{31}$. In this cell, both the plates and the lateral walls was rough and the authors report a $N u \propto R a^{1 / 2}$ scaling law consistent with fully turbulent heat transfers. We fit Eq. 26 in the least-square sense, in the range of $\operatorname{Pr}$ close to ours. We find $\sigma=0.065$ (see figure 9). This value, fitted in a turbulent rough cell, is close to $\sigma=0.06 \mathrm{usu}-$ ally found in the literature for smooth cells at moderate Rayleigh numbers.

However, the reduced Nusselt numbers, $n$, that we obtain in our cell, although larger than the smooth case one, are still about 1.5 times smaller than the reduced Nusselt numbers computed from the data of Roche, et al.. Additionnally, the observations show a thick boundary layer inside the notches. This is not consistent with a fully turbulent boundary layer.

\section{Turbulent BL on the top of the plots}

In the range of parameters that we can achieve in our rectangular cell, it is possible that only the boundary layers at the top of the plots are destabilized. Since the

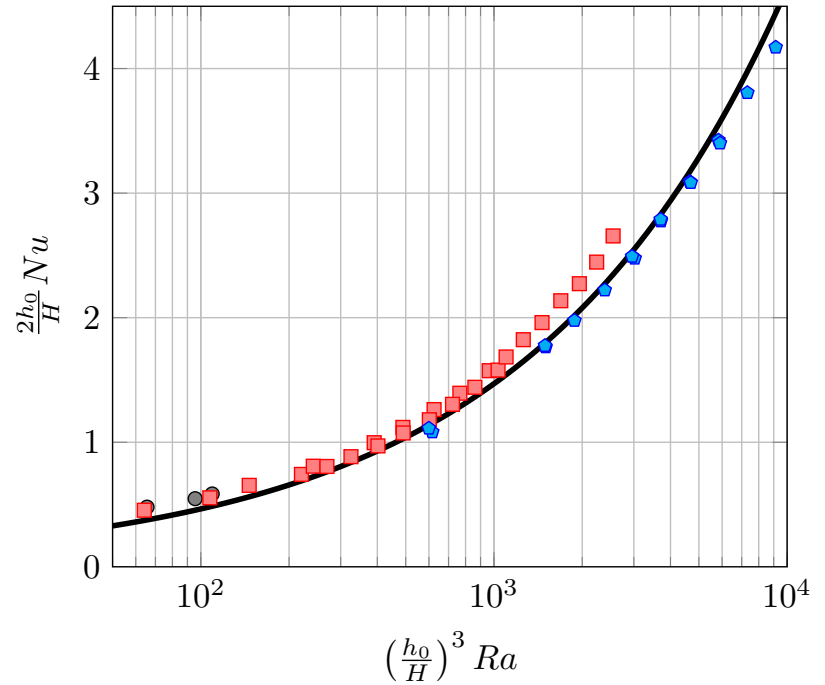

FIG. 9. Heat transfer measurements obtained by Roche, et al. in a rough cell ${ }^{31}$. Circles: $\operatorname{Pr}=1$. Squares: $\operatorname{Pr}=2.7$. Pentagons: $\operatorname{Pr}=5.6$. Solid line: Eq. 26 with $\sigma=0.065$.

plots and the notches both occupy one quarter of the plate surface, the rough Nusselt number will be given by

$$
N u_{r}=\frac{1}{2} N u_{\text {groove }}+\frac{1}{4} N u_{\text {notch }}+\frac{1}{4} N u_{\text {plot }}
$$

To test this interpretation, we have to derive each contribution.

We assume that the boundary layers on the top of the plots are turbulent. Therefore, $N u_{\text {plot }}$ will be given by a relation similar to Eq. 23:

$$
N u_{\text {plot }}=\frac{(2 \sigma)^{3 / 2}}{2}\left(\frac{h_{0}}{H}\right)^{1 / 2} R a^{1 / 2}
$$

The fluid in the notches is almost quiet, and the boundary layer thickness is large. To take into account the advection that may still occur inside the notch, we can compute the Rayleigh number, $R a_{h_{0}}$, of this small convection cell of height $h_{0}$,

$$
R a_{h_{0}}=\left(\frac{h_{0}}{H}\right)^{3} \frac{T_{h}-T_{\mathrm{bulk}}}{T_{h}-T_{c}} R a
$$

If we call $f$, the function that gives the Nusselt number for a given Rayleigh number in the case of a smooth cell, then the heat flux in the notch, $Q_{\text {notch }}$ is given by

$$
\frac{Q_{\text {notch }} h_{0}}{\lambda\left(T_{h}-T_{\text {bulk }}\right)}=f\left(\left(\frac{h_{0}}{H}\right)^{3} \frac{T_{h}-T_{\text {bulk }}}{T_{h}-T_{c}} R a\right)
$$

From this equation, we can compute the Nusselt number associated to the notches,

$$
N u_{\text {notch }}=\frac{Q_{\text {notch }} H}{\lambda\left(T_{h}-T_{c}\right)}
$$


We can neglect the fact that $T_{\text {bulk }}$ is larger than $\left(T_{h}+\right.$ $\left.T_{c}\right) / 2$ and write

$$
\frac{T_{h}-T_{\text {bulk }}}{T_{h}-T_{c}} \approx \frac{1}{2}
$$

Thus, using the heat flux from Eq. 30,

$$
N u_{\text {notch }}=\frac{H}{2 h_{0}} f\left(\frac{1}{2}\left(\frac{h_{0}}{H}\right)^{3} R a\right)
$$

At moderate Rayleigh numbers, the smooth Nusselt numbers, $N u_{s}=f\left(R a_{s}\right)$ that we measure in the smooth half cell fall fairly close to the prediction of the Grossmann-Lohse model ${ }^{32}$ with the recently updated prefactors $^{33}$. Therefore, we will use the GL model for the function $f$. On the other hand, the Rayleigh number of the notch is much smaller, close to the onset of convection. In this range of low Rayleigh numbers, the GL model cannot be used. Therefore, we use a linear interpolation of experimental data from Chavanne, et al. ${ }^{34}$. In this range of parameters, the data from Chavanne, et $a l$. is in fair agreement with all other published data. We can rewrite Eq. 34 more explicitly using $f_{G L}$, the function computed from the GL model, and $f_{C}$, the function computed from Chavanne, et al. experimental data :

$N u_{\text {notch }}= \begin{cases}f_{G L}(R a) & \text { when } N u<H /\left(2 h_{0}\right) \\ \frac{H}{2 h_{0}} f_{C}\left(\frac{1}{2}\left(\frac{h_{0}}{H}\right)^{3} R a\right) & \text { when } N u>H /\left(2 h_{0}\right)\end{cases}$

To estimate the final rough Nusselt number, $N u_{r}$, we also have to compute a local Nusselt number for the grooves. We assume that they behave as a smooth plate, but we take into account the contribution of the vertical walls, i.e., the surface increase can be written, using again $f_{G L}$ :

$$
\frac{S_{\text {groove }}}{S_{\text {smooth }}}=\frac{2 \ell^{2}+2\left(h_{0}-\delta_{t h}\right) \ell}{2 \ell^{2}}=1+\frac{h_{0}}{\ell}-\frac{H}{2 \ell f_{G L}(R a)}
$$

and therefore

$$
N u_{\text {groove }}=\left(1+\frac{h_{0}}{\ell}\right) f_{G L}(R a)-\frac{H}{2 \ell}
$$

The expected rough Nusselt numbers are obtained by combining Eq. 27 with Eqs. 28, 34, 36. This system has no free parameters : $\sigma=0.065$ was fitted from the experimental data of Roche, et al., the function $f_{C}$ is obtained from the experimental data of Chavanne, et al. at low Rayleigh numbers and $f_{G L}$ is obtained from the GL model at moderate Rayleigh numbers. A comparison between this model and the heat-transfer measurements previously shown in figure 2 , and also the heat-transfer measurements of Tisserand, et al. carried out in two cylindrical cells ${ }^{15}$ of aspect ratios $\Gamma=0.5$ and $\Gamma=2.5$, are shown in figure 10 .

For reference, the smooth data and the GL model is also plotted in figure 10. Note that it is possible to plot the smooth data with the same reduced variables as the rough case if the $N u$ scales like $R a^{1 / 3}$, indeed $N u=$ $0.06 R a^{1 / 3}$ can written with the rough reduced variables :

$$
n=0.12 r^{1 / 3}
$$

So, one advantage of these reduced variables is that the two limit cases (roughness-induced turbulent case $N u \propto$ $\sqrt{h_{0} / H} R a^{1 / 2}$, and the regular smooth $N u \propto R a^{1 / 3}$ case) should collapse on curves that both no longer depend on the roughness height $h_{0}$.

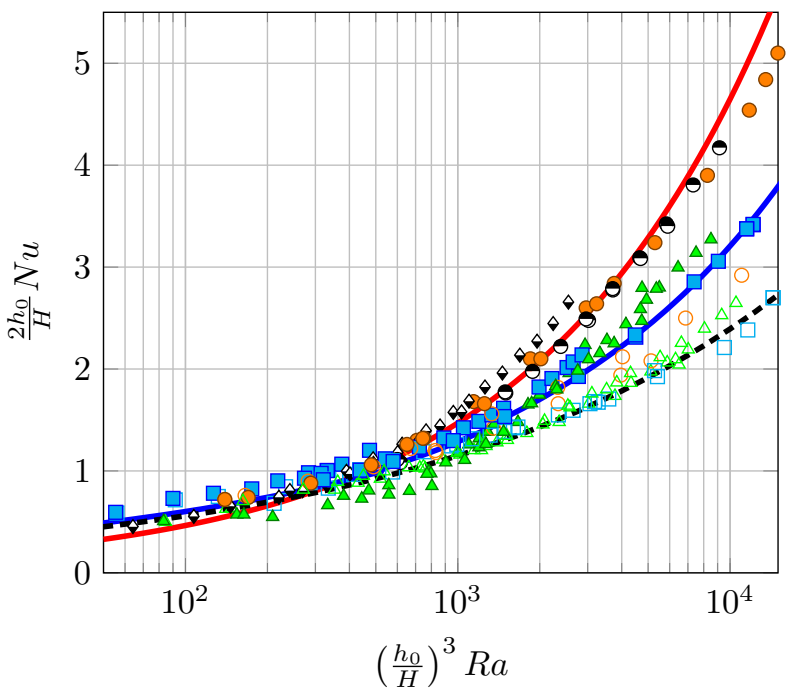

FIG. 10. Experimental heat-transfer data in reduced variables. Full symbols: rough Nusselt numbers. Open symbols: smooth Nusselt numbers. Squares: present measurements. Triangles and Circles: respectively, the "Tall cell" and the "Small cell" of Tisserand, et al. ${ }^{15}$. Half-filled symbols: results of Roche, et al. in a fully turbulent rough cell ${ }^{31}$ (diamond: $\operatorname{Pr}=2.7$, circle: $\operatorname{Pr}=5.6$ ). Dashed line: theoretical prediction for a smooth cell with the GL model. Upper solid line: $n=(2 \sigma)^{3 / 2} r^{1 / 2}$. Lower solid line: prediction from Eq. 27 .

\section{E. Discussion}

The present heat-transfer data is in good agreement with the model prediction based on the assumption that the BL is turbulent on the top of the plots only. The data from Tisserand, et al. "Tall cell" (cylindrical cell of height $H=1 \mathrm{~m}$ ) coincides well with the present data and predictions.

However, the measurements from Tisserand, et al "Small cell" (cylindrical cell of height $H=20 \mathrm{~cm}$ ) is much closer to the fully turbulent data of Roche, et al. and the turbulent prediction $n=(2 \sigma)^{3 / 2} r^{1 / 2}$ (see figure $10)$. The reason may be that the mean velocity is larger in the "Small cell" than in the "Tall cell", which leads to larger Reynolds numbers, and therefore possibly to a fully turbulent BL. 
To test further the agreement between the model and our experimental observations, we can estimate the boundary layer thicknesses, $\delta_{\text {plot }}=H /\left(2 N u_{\text {plot }}\right)$, and $\delta_{\text {notch }}=H /\left(2 N u_{\text {notch }}\right)$. The resulting boundary layer thicknesses are plotted in figure 11. In particular, for a smooth boundary layer thickness of order $0.8 \mathrm{~mm}$, the corresponding boundary layer thickness above a plot is $0.4 \mathrm{~mm}$ and the boundary layer thickness inside the notch is $1.8 \mathrm{~mm}$. This is in fair agreement with our experimental data.

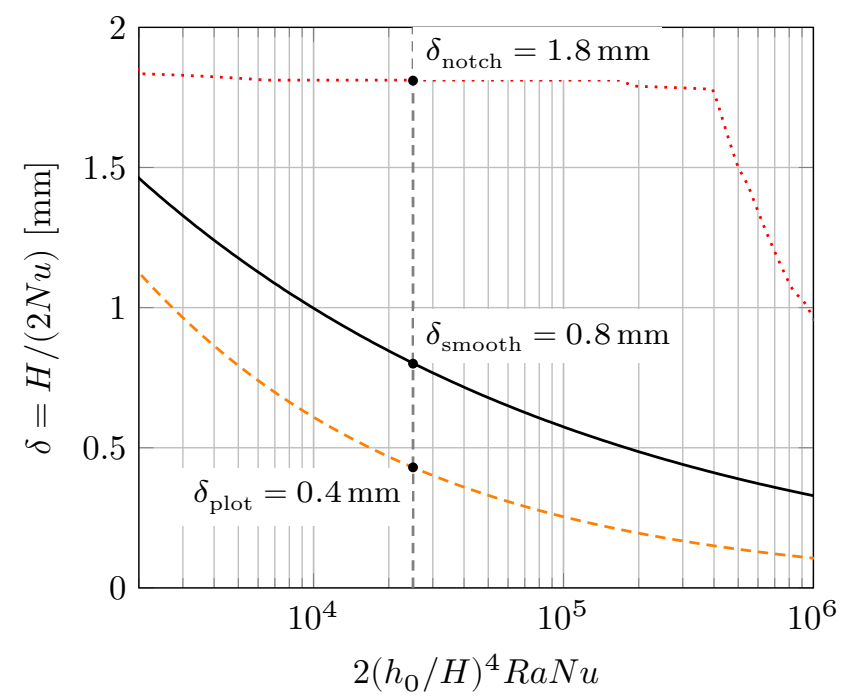

FIG. 11. Boundary layer thickness above a plot (dashed line), and inside a notch (dotted line) compared to the boundary layer thickness of a smooth plate (solid line) computed respectively with our local Nusselt number model (Eq. 28 and Eq. 34) and with the Grossmann-Lohse model

Although the roughness geometries are different, our result and our interpretation can be compared to the measurements of $\mathrm{Du}$ and $\mathrm{Tong}^{21}$. In their case, the cell has rough upper and lower surfaces, with pyramidal roughnesses. In this cell, they obtain an enhanced heat transfer but with no change in the $N u$ versus $R a$ scaling: the scaling is always $R a^{2 / 7}$ but the power-law amplitude is increased by more than $76 \%$. For reference, we shall also cite the work of Qiu, Xia and Tong ${ }^{14}$ which reports a $N u \sim R a^{0.35}$ scaling law. This exponent, although larger than $1 / 3$ is closer to the laminar case.

These differences between those results and ours can be the consequence of the shape of the roughnesses. Indeed, in their case, only the tip of the roughness elements is inside the bulk region. In our case, the top of the roughness elements consists in a $5 \mathrm{~mm}$-long flat zone where a boundary layer has enough room to form. Therefore, the behavior of the boundary layer may be different. In their case, they showed that the pyramidal roughnesses enhance the plume emissions. In our setup, we observe also enhancements of plume emissions, but only intermittenly, as will be discussed at the end of section IV.
Yet our results also bear some similarities with theirs. The enhancement is greater than the surface increase, induced by the roughnesses. They have seeded their flow with thermochromic liquid crystal spheres, which allows velocity and temperature visualization. They clearly evidence secondary flows in the groove region, which is consistent with our notch Rayleigh number that is found larger than the convection threshold, as was also previously pointed out by Tisserand, et al. ${ }^{15}$. In addition, they also find a much larger thermal boundary layer thickness inside the groove and a much thinner one at the tip of the rough element, which is consistent with our observations.

One possible interpretation is that the intermediate regime where only the boundary layers at the top of the roughness become turbulent is not accessible with pointy roughnesses. If the Reynolds number is large enough, a fully turbulent regime can be observed, as in the Roche, et al. case $^{31}$, leading to a change in the heat-transfer scaling law. If it is not, then the boundary layer does not become turbulent and the scaling law is not changed. Yet, heat-transfer enhancement is still possible with to other mechanisms, such as enhancement of plume emissions, as in the Du and Tong case ${ }^{21}$.

\section{MULTISTABILITY AND LONGER TIME SCALES}

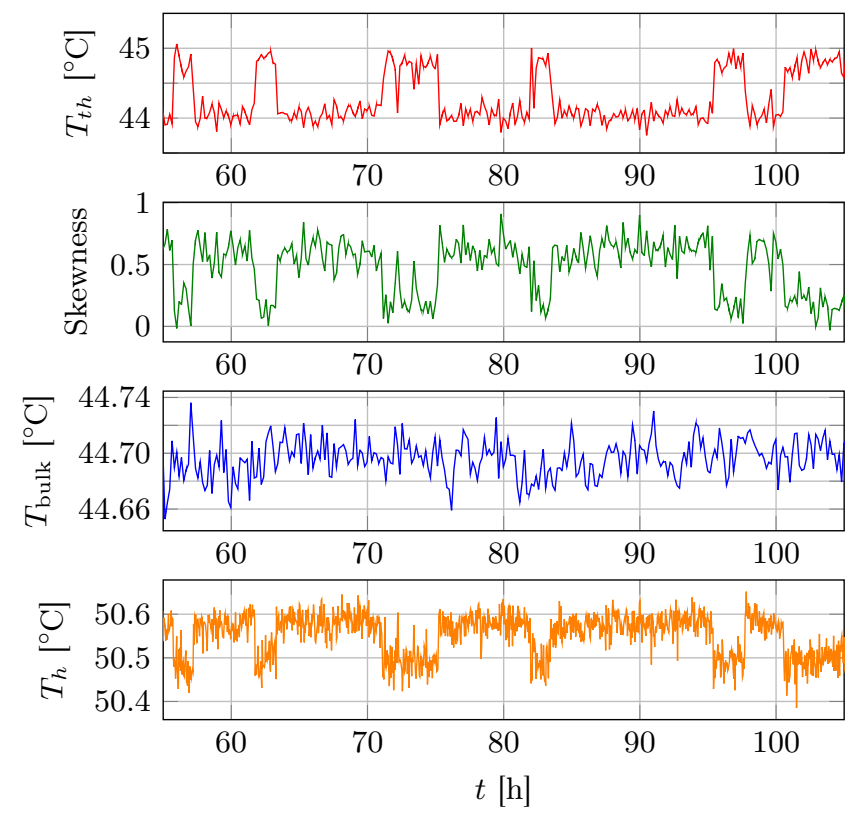

FIG. 12. Example of coarse-grained timeseries showing evidence of multistability in the flow. From top to bottom: signal of a thermistor located inside a notch at $z=0.95 \mathrm{~mm}$, skewness of the thermistor signal computed on a moving window, signal of a second thermistor located in the bulk and signal of a PT100 sensor on the left-hand-side of the bottom plate.

We have recorded long time series of the thermistor 
signal at various positions inside the cell. An example of coarse-grained time series is plotted in figure 12. The signal exhibits transitions between two states: in the following, we shall call "high-Nu" the state where $T_{h}$ is colder (because $\Delta T=T_{h}-T_{c}$ is smaller and therefore $N u$ is larger), and "low-Nu" the state where $T_{h}$ is warmer. This additionnal increase of Nusselt number, of order $1 \%$, is much smaller to the one described in the previous section. The time scales associated with these transitions are much longer than the typical turnover time.

It is well known that the mean flow in the RayleighBénard system can display large scale multistability, eg. reversals $^{35-37}$ or changes in the convective pattern ${ }^{38,39}$. These transitions are known to exhibit time scales much longer than all other typical times. This type of behavior is also reported in other turbulent closed flows, such as Von Kármán flow ${ }^{40}$.

In the present observation, there were no reversals during the time of the acquisition. So this multistability is not caused by a flow reversal. There is also no measurable signature in the bulk temperature, nor in temperature signals near the top plate nor in signal of the PT100 inside top plate. However, the transitions evidenced by the thermistor inside the notch are correlated to changes in the bottom plate temperature, and therefore to changes in the Nusselt number. It is worth noting that bistability of the Nusselt number has been reported at moderate Rayleigh numbers in high-precision cryogenic helium convection cells ${ }^{41-43}$, and also in water by Chillà, et al. ${ }^{44}$.

The statistics of the thermistor signal at this particular position in the notch differs between the two states: the distribution, shown in figure 13, is more skewed in the "low-Nu" state, in this case. We have recorded time series at various positions in several notches, as well as in grooves, and found out that there is a simultaneous change of statistics everywhere near the rough plate. However, the effects of this transition on the statistics highly depend on the exact position of the thermistor. There are positions where the thermistor temperature signal step up when the plate temperature gets higher, unlike the signal shown in figure 12. Therefore, the only robust conclusion that can be drawn, is that these two states are associated with a change in the structure of the flow, but it is not clear how the boundary layer changes. This change is visible simultaneously in notches and in grooves, even if the probes are spacially separated (an example is given in figure 14). This means that the change of flow structure affects large areas of the bottom plate.

To figure out the nature of the two regimes, we reconstructed the field of the temperature fluctuations with an optical technique based on the light refraction. Indeed, the temperature gradients in the fluid induce a gradient of optical index. These optical index gradients can be measured optically. This kind of measurements was first successfully used for temperature imaging in RayleighBénard convection using a laser scanning technique ${ }^{45,46}$. In the present work, we combined this technique with the modern, more straightforward, computer-based method
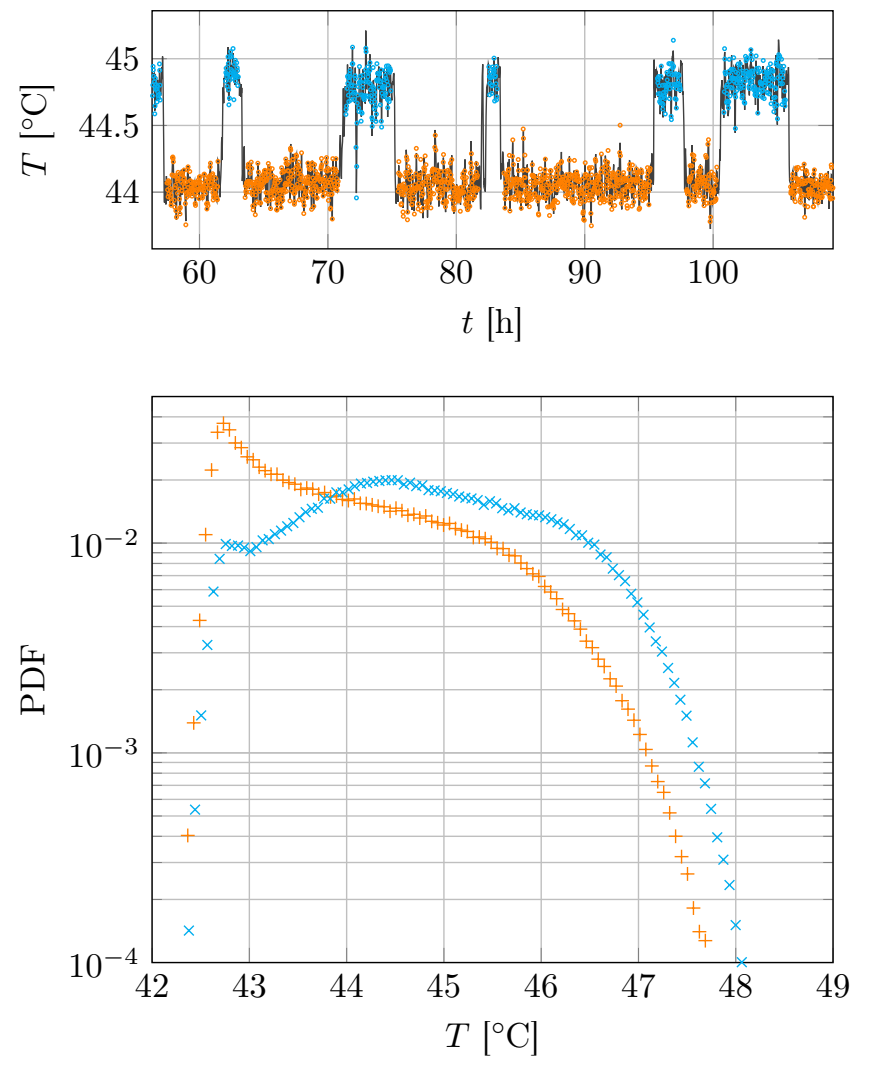

FIG. 13. Conditionnal analysis of the time series shown in figure 12 , recorded inside a notch at $z=0.95 \mathrm{~mm}$. Top: thermistor coarse-grained time series devided into two states based on a threshold on the plate temperature from the PT100. Bottom: conditionnal histogram computed on the "High-Nu" state (x crosses) data points and on the "low-Nu" state (+ crosses).

of background-oriented synthetic Schlieren ${ }^{47,48}$. Unlike shadowgraphy, this more quantitative technique grants access to the temperature field, and thus to statistical quantities.

The background picture is a computer-generated image of randomly spaced dots. The region of interest, shown as a brown dashed area in figure 1 was recorded with a black and white AVT Stingray F125B camera. The framerate is one image every five seconds during up to a hundred hours, with synchroneous acquisition of the thermistors. The apparent motion of the dots in the background pattern is computed using a cross-correlation algorithm implemented in the CIVx software suite ${ }^{49}$. The vector field, $\vec{d}(x, z, t)$, computed from the image at time $t$ and a reference image obtained before the experiment starts, is

$$
\vec{d}(x, z, t) \propto \nabla \rho(x, z, t) \propto \nabla T(x, z, t)
$$

However, in our system, gradients of optical index also exist inside the plexiglas walls. This prevented us from getting a reference image of the background pattern with the fluid at rest (the temperature difference between the 

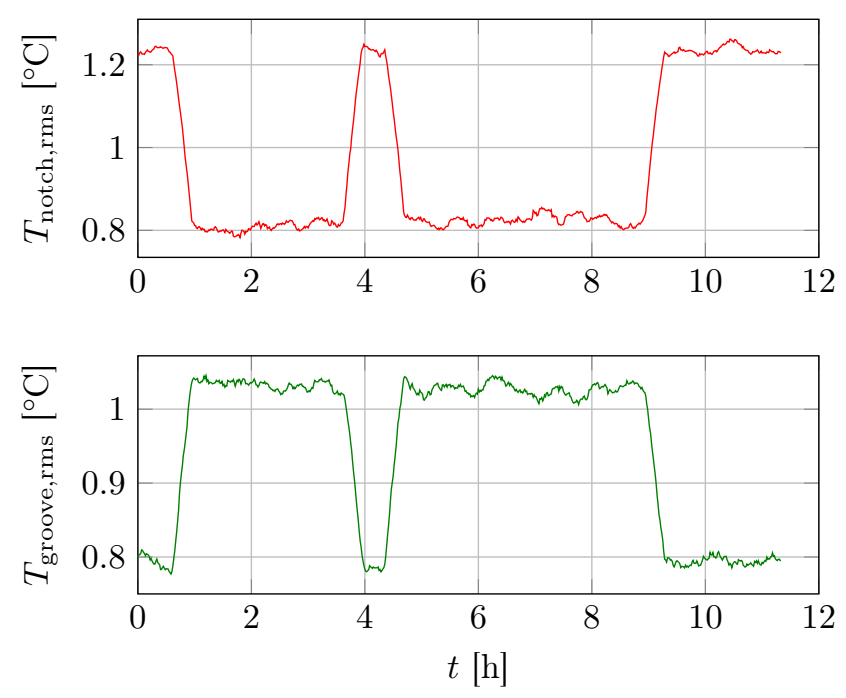

FIG. 14. Example of simultaneous acquisitions from two thermistors inside the cell. Top: rms of the temperature fluctuations inside a notch at $z=0.70 \mathrm{~mm}$. Bottom: rms of the thermistor in a groove approximately $10 \mathrm{~cm}$ away from the first thermistor.

bottom and the top of the wall leads to a spurious dot motion). Therefore, we had to use cross-correlations between two successive images instead. The computed vector field, $\vec{d}_{\tau}(x, z, t)$ is therefore

$$
\vec{d}_{\tau}(x, z, t)=\vec{d}(x, z, t+\tau)-\vec{d}(x, z, t)
$$

with $\tau=5 \mathrm{~s}$ in our experiment. After integration in space of this vector field, we obtain a scalar field, $T_{\tau}(x, z, t)$,

$$
T_{\tau}(x, z, t) \propto T(x, z, t+\tau)-T(x, z, t)
$$

In the following, $\langle$.$\rangle means average in time, T_{0}(x, z)=$ $\langle T(x, z, t)\rangle$ and $\delta T(x, z, t)=T(x, z, t)-T_{0}(x, z)$. Obviously, $\left\langle T_{\tau}\right\rangle$ is zero everywhere. We note $T_{\tau, \mathrm{rms}}(x, z)=$ $\sqrt{\left\langle T_{\tau}(x, z, t)^{2}\right\rangle}$. It is given by,

$T_{\tau, \mathrm{rms}}(x, z)^{2} \propto\left\langle\delta T(x, z, t)^{2}\right\rangle-\langle\delta T(x, z, t+\tau) \delta T(x, z, t)\rangle$

If $\tau$ is sufficiently large, the cross-correlation term $\langle\delta T(x, z, t+\tau) \delta T(x, z, t)\rangle$ can be neglected, and thus $T_{\tau, \mathrm{rms}}$ is proportional to the rms temperature fluctuations.

Figure 15 shows two $T_{\tau, \mathrm{rms}}(x, z)$ fields with similar color codes, one is averaged in the "low-Nu" state, the other in the "high-Nu" state. They show qualitative difference between the two regimes. The latter is much more agitated that the former (there are wide areas with a larger value of the temperature fluctuations rms). A slice is shown in figure 16 to better evaluate the increase.

Our interpretation is that there is another mechanism that adds up to the emergence of turbulence discussed in section III: the enhancement of plume emissions. This sort of effect has been reported for pyramidal roughnesses by Du and Tong ${ }^{21}$, and this effect also induces wider temperature distributions, at least in the center of the cell, in their case ${ }^{12}$. In our cell, the "High $\mathrm{Nu}$ " state presents intermittent bursts of coherent plumes. Example of this kind of bursts is shown in figure 17. These bursts occur only infrequently, typically once or twice every minute, and each burst lasts typically five to ten seconds. This phenomenon leads to an increase of the Nusselt number only of order $1 \%$, much lower than the increase reported by Du and Tong. It is not clear if the enhancement of plume emissions that we report is similar to theirs, because our observations are at much larger scales. We do not have information on the details of the plume emission enhancement very close to the roughnesses at the moment. A possible reason for the lower Nusselt increase is the infrequent nature of the burst emissions, that may depend on the details of the roughness geometry. Note that the emissions of coherent plumes have also been evidenced at very high Rayleigh numbers, beyond the transition threshold to turbulent boundary layers, by Gauthier, et al. ${ }^{7}$ from the careful analysis of the plate temperature fluctuations.

\section{v. DISCUSSION}

The transition to turbulence of the boundary layer is an important problem for Rayleigh-Bénard convection. Theoretical works have predicted that this transition, at asymptotically large Rayleigh numbers, leads to a change in the heat transfer scaling law ${ }^{30,50}$. This enhanced heat transfer regime is sometimes referred to as "ultimate regime of convection". Several groups have reported a transition to an enhanced heat transfer regime with an experimental critical Rayleigh number varying between $10^{12}$ for the Grenoble results ${ }^{8}$ and $10^{14}$ for the Göttingen results $^{51}$. The problem of critical transition values in this kind of turbulent closed system is well known to highly depend on details of the boundary conditions ${ }^{52,53}$. A key problem is therefore to figure out if it is possible to purposely change the boundary conditions so that the transition to turbulence is triggered at moderate Rayleigh numbers. One approach consists in adding controlled roughnesses and study how they interact with the flow.

In the present work, heat transfer enhancement is reported when the boundary layer thickness matches the height of the roughnesses. This is consistent with previous global heat transfer measurements ${ }^{10,11,14,15}$. Systematic characterizations of the temperature statistics near the boundaries have been carried out in the enhanced regime. They indicate, in the range of parameters explored, that the fluid inside the notches is essentially at rest and that the boundary layer on the plot is very thin: this seems to explain most of the enhancement of the heat transfer. The boundary layer on top of the plots is destabilised by the mean flow.

We also report intermittent transitions to a more ag- 

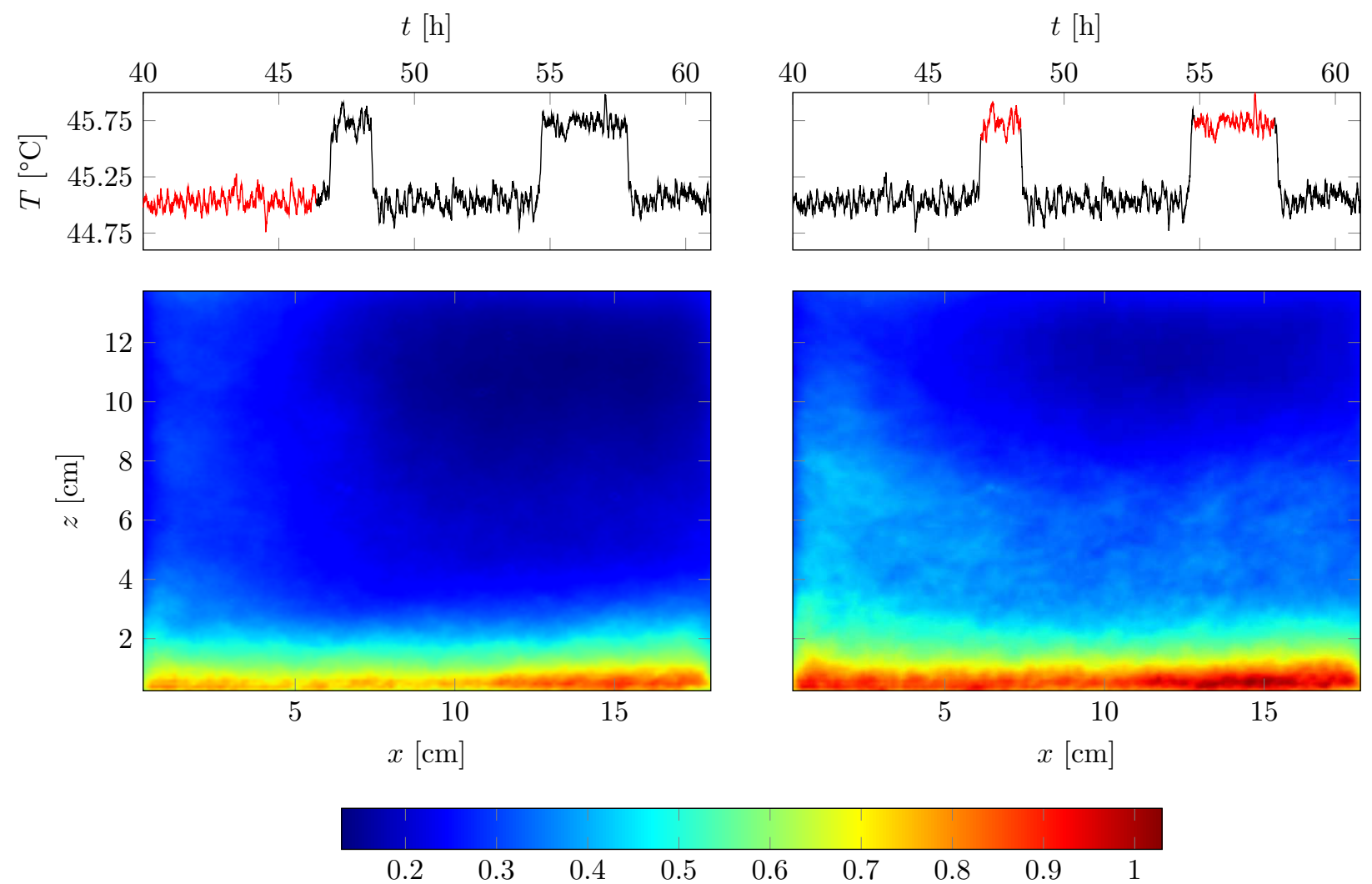

FIG. 15. Top: temperature signal of the thermistor located in a notch. The red part of the time series indicates the time intervals where the average is computed. Bottom: $T_{\tau, \mathrm{rms}}(x, z)$ obtained with background-oriented synthetic Schlieren (see text). The number of images used to compute the average is nearly the same for both images to prevent statistical bias. Left: picture obtained in the "low-Nu" regime. Right: picture obtained in the "high-Nu" regime. The picture color code is similar for both images.

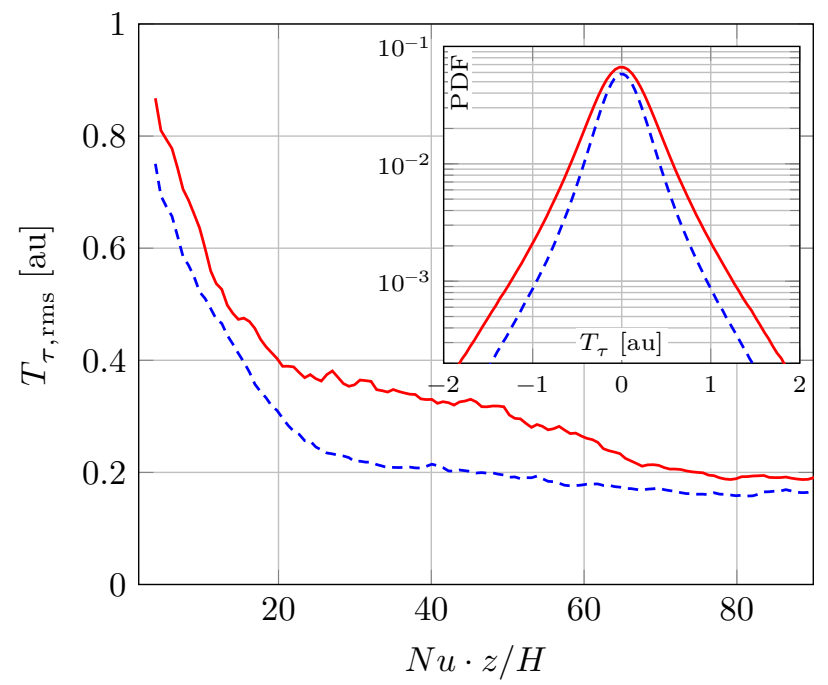

FIG. 16. Slice of the $T_{\tau, \mathrm{rms}}(x, z)$ fields shown in figure 15 at $x=8.1 \mathrm{~cm}$. Inset: probability density function of $T_{\tau}(x, z, t)$ in both regimes. Solid red line: "High-Nu" regime. Dashed blue line: "Low-Nu" regime.
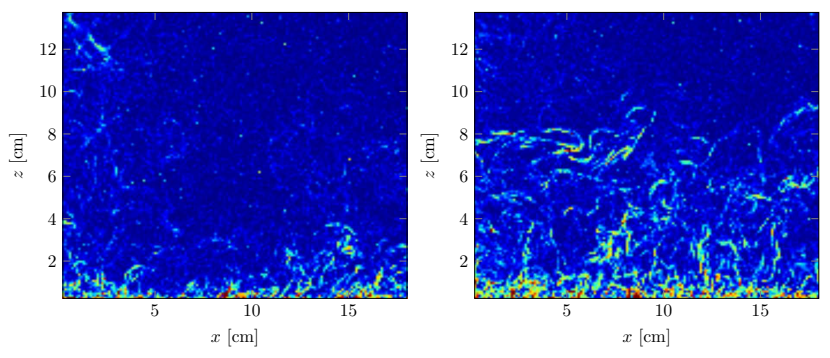

FIG. 17. Instantaneous module of dot displacement, with the same color code (blue is no displacement, red is 1.6 pixels). Left: Typical field in the "Low Nu" state. Right: Example of plume burst in the "High $\mathrm{Nu}$ " state.

itated state associated with a higher Nusselt number. This additionnal increase is much smaller (of order 1\%), in the range of parameters that we can investigate in this cell. Our interpretation is that this is caused by another mechanism, maybe close to the $\mathrm{Du}$ and Tong scenario: the enhancement of plume emissions by the roughnesses. Indeed, we have observed intermittent burst of coherent plumes in this more agitated state. 
Various observations reported in the literature on rough Rayleigh-Bénard systems, with pyramids ${ }^{9,10,14,21}$, V-shape grooves ${ }^{18,31}$, or square-studs ${ }^{15}$ show both similarities and apparent contradictions. They all report heat transfer enhancement when the thermal boundary layer thickness matches the height of the roughness, larger than the surface increase, which suggests that the triggering of some enhancement mechanisms. But the amount of increase can be very different: some have reported no change in the scaling law but a change in prefactor, some have reported a transition to a fully turbulent $R a^{1 / 2}$ scaling laws, or intermediate cases with an increase of the scaling law exponent. In our cell, we observe the effect of two different mechanisms: the emergence of turbulence and the enhancement of plume emissions. This can explain the apparent discrepancy between the published results.

Depending on the roughness geometry, and on the mean velocity, the critical shear Reynolds number can be lowered, not enough to destabilize the boundary layer (Du and Tong case), just enough to destabilize the top of the roughness elements (our case) or sufficiently to obtain a fully turbulent boundary layer (Roche, et al. case).

\section{ACKNOWLEDGMENTS}

We thank Marc Moulin and Denis Le Tourneau for technical assistance, and Artyom Petrosyan and Hélène Scolan for useful discussions on optics and on the background-oriented synthetic Schlieren technique. The data analysis has been partially achieved thanks to the resources of PSMN (Pôle Scientifique de Modélisation Numérique) of ENS Lyon. This work has been partly supported by the Région Rhône Alpes (Cible 2011, $\left.\mathrm{n}^{\circ} 2770\right)$.

${ }^{1}$ L. Rayleigh, "On convection currents in a horizontal layer of fluid, when the higher temperature is on the underside," Philosophical Magazine 32, 529-546 (1916).

${ }^{2}$ F. Chillà and J. Schumacher, "New perspectives in turbulent rayleigh-bénard convection," Eur. Phys. J. E 35, 58 (2012).

${ }^{3}$ F. Chillà, M. Rastello, S. Chaumat, and B. Castaing, "Ultimate regime in rayleigh-bénard convection: The role of plates," Physics of Fluids 16, 2452-2456 (2004).

${ }^{4} \mathrm{R}$. Verzicco, "Effects of nonperfect thermal sources in turbulent thermal convection," Physics of Fluids 16, 1965-1979 (2004).

${ }^{5}$ E. Brown, A. Nikolaenko, D. Funfschilling, and G. Ahlers, "Heat transport in turbulent rayleigh-bénard convection: Effect of finite top- and bottom-plate conductivities," Physics of Fluids 17, 075108 (2005).

${ }^{6}$ R. Verzicco and K. R. Sreenivasan, "A comparison of turbulent thermal convection between conditions of constant temperature and constant heat flux," Journal of Fluid Mechanics 595, 203219 (2008).

${ }^{7}$ F. Gauthier and P.-E. Roche, "Evidence of a boundary layer instability at very high rayleigh number," EPL 83, 24005 (2008).

${ }^{8}$ P.-E. Roche, F. Gauthier, R. Kaiser, and J. Salort, "On the triggering of the ultimate regime of convection," New J. Phys. 12, 085014 (2010).

${ }^{9}$ Y. Shen, P. Tong, and K.-Q. Xia, "Turbulent convection over rough surfaces," Phys. Rev. Lett. 76, 908 (1996).
${ }^{10}$ Y.-B. Du and P. Tong, "Enhanced heat transport in turbulent convection over a rough surface," Phys. Rev. Lett. 81, 987-990 (1998).

${ }^{11} \mathrm{~S}$. Ciliberto and C. Laroche, "Random roughness of boundary increases the turbulent convection scaling exponent," Phys. Rev. Lett. 82, 3998 (1999).

${ }^{12}$ Y.-B. Du and P. Tong, "Temperature fluctuations in a convection cell with rough upper and lower surfaces," Phys. Rev. E 63, 046303 (2001).

${ }^{13}$ P.-E. Roche, B. Castaing, B. Chabaud, and B. Hébral, "Heat transfer in turbulent Rayleigh-Bénard convection below the ultimate regime," J. Low Temp. Phys. 134, 1011-1042 (2004).

${ }^{14}$ X.-L. Qiu, K.-Q. Xia, and P. Tong, "Experimental study of velocity boundary layer near a rough conducting surface in turbulent natural convection," Journal of Turbulence 6, 1 (2005).

${ }^{15}$ J.-C. Tisserand, M. Creyssels, Y. Gasteuil, H. Pabiou, M. Gibert, B. Castaing, and F. Chillà, "Comparison between rough and smooth plates within the same rayleigh-bénard cell," Phys. fluids 23, 015105 (2011).

${ }^{16} \mathrm{O}$. Shishkina and C. Wagner, "Modelling the influence of wall roughness on heat transfer in thermal convection," J. Fluid Mech. 686, 568-582 (2011).

${ }^{17}$ A. García, J. P. Solano, P. G. Vicente, and A. Viedma, "The influence of artificial roughness shape on heat transfer enhancement: Corrugated tubes, dimpled tubes and wire coils," Applied Thermal Engineering 35, 196-201 (2012).

${ }^{18}$ G. Stringano, G. Pascazio, and R. Verzicco, "Turbulent thermal convection over grooved plates," J. Fluid Mech. 557, 307-336 (2006).

19 A. Tilgner, A. Belmonte, and A. Libchaber, "Temperature and velocity profiles of turbulent convection in water," Phys. Rev. E 47, R2253-R2256 (1993).

${ }^{20}$ A. Belmonte, A. Tilgner, and A. Libchaber, "Temperature and velocity boundary layers in turbulent convection," Phys. Rev. E 50, 269-279 (1994).

${ }^{21}$ Y.-B. Du and P. Tong, "Turbulent thermal convection in a cell with ordered rough boundaries," J. Fluid Mech. 407, 57-84 (2000).

${ }^{22}$ R. du Puits, C. Resagk, A. Tilgner, F. Busse, and A. Thess, "Structure of thermal boundary layers in turbulent rayleighbénard convection," J. Fluid Mech. 572, 231-254 (2007).

${ }^{23}$ Q. Zhou and K.-Q. Xia, "Thermal boundary layer structure in turbulent rayleigh-bénard convection in a rectangular cell," J. Fluid Mech. 721, 199-224 (2013).

${ }^{24}$ R. du Puits, C. Resagk, and A. Thess, "Thermal boundary layers in turbulent rayleigh-bénard convection at aspect ratios between 1 and 9," New J. Phys. 15, 013040 (2013).

${ }^{25}$ The value of the prefactor can be found ${ }^{54}$ by integrating numerically the ordinary differential equation $2 f^{\prime \prime \prime}+f f^{\prime \prime}=0$.

${ }^{26}$ The value of the prefactor can be found ${ }^{54}$ by integrating numerically the ordinary differential equation $T^{\prime \prime}+\frac{\zeta^{2}}{4} T^{\prime}=0$.

${ }^{27}$ W. L. Shew, Y. Gasteuil, M. Gibert, P. Metz, and J.-F. Pinton, "Instrumented tracer for lagrangian measurements in rayleighbénard convection," Rev. Sci. Instrum. 78, 065105 (2007).

${ }^{28}$ L. Li, N. Shi, R. du Puits, C. Resagk, J. Schumacher, and A. Thess, "Boundary layer analysis in turbulent rayleigh-bénard convection in air: Experiment versus simulation," Phys. Rev. E 86, 026315 (2012).

${ }^{29}$ H. Schlichting, Boundary-Layer Theory, 8th ed. (Springer, New York, 2000).

${ }^{30}$ R. H. Kraichnan, "Turbulent thermal convection at arbitrary prandtl number," Phys. fluids 5, 1374-1389 (1962).

${ }^{31}$ P.-E. Roche, B. Castaing, B. Chabaud, and B. Hébral, "Observation of the $1 / 2$ power law in rayleigh-bénard convection," Phys. Rev. E 63, 045303(R) 1-4 (2001).

${ }^{32} \mathrm{~S}$. Grossmann and D. Lohse, "Prandtl and rayleigh number dependence of the reynolds number in turbulent thermal convection," Phys. Rev. E 66, 016305 (2002).

${ }^{33}$ R. J. A. M. Stevens, E. P. van der Poel, S. Grossmann, and D. Lohse, "The unifying theory of scaling in thermal convection: 
The updated prefactors," J. Fluid Mech. 730, 295-308 (2013).

${ }^{34} \mathrm{X}$. Chavanne, F. Chillà, B. Chabaud, B. Castaing, and B. Hébral, "Turbulent rayleigh-bénard convection in gaseous and liquid he," Phys. fluids 13, 1300-1320 (2001).

${ }^{35}$ K. R. Sreenivasan, A. Bershadskii, and J. J. Niemela, "Mean wind and its reversal in thermal convection," Phys. Rev. E 65, 056306 (2002).

${ }^{36} \mathrm{E}$. Brown and G. Ahlers, "Rotations and cessations of the largescale circulation in turbulent rayleigh-bénard convection," Journal of Fluid Mechanics 568, 351-386 (2006).

${ }^{37}$ H.-D. Xi and K.-Q. Xia, "Cessations and reversals of the largescale circulation in turbulent thermal convection," Phys. Rev. E 75, 066307 (2007).

${ }^{38}$ S. Ciliberto, E. Pampaloni, and C. Pérez-García, "Competition between different symmetries in convective patterns," Phys. Rev. Lett. 61, 1198 (1988).

${ }^{39}$ E. Bodenschatz, J. R. de Bruyn, G. Ahlers, and D. S. Cannell, "Transitions between patterns in thermal convection," Phys. Rev. Lett. 67, 3078 (1991).

${ }^{40}$ P.-P. Cortet, E. Herbert, A. Chiffaudel, F. Daviaud, B. Dubrulle, and V. Padilla, "Susceptibility divergence, phase transition and multistability of a highly turbulent closed flow," J. Stat. Mech. , 07012 (2011).

${ }^{41}$ D. C. Threlfall, "Free convection in low-temperature gaseous helium," J. Fluid Mech. 67, 17-28 (1975).

${ }^{42}$ P.-E. Roche, B. Castaing, B. Chabaud, and B. Hébral, "Prandtl and Rayleigh numbers dependences in Rayleigh Bénard convection," Europhys. Lett. 58, 693-698 (2002).

${ }^{43}$ J. Salort, F. Gauthier, B. Chabaud, O. Bourgeois, J.-L. Garden, R. du Puits, A. Thess, and P.-E. Roche, "Convection at very high rayleigh number: signature of transition from a microthermometer inside the flow," in Advances in Turbulence XII, Springer Proceedings in Physics, Vol. 132 (2009) pp. 159-162, hal-00430188.
${ }^{44}$ F. Chillà, M. Rastello, S. Chaumat, and B. Castaing, "Long relaxation times and tilt sensitivity in rayleigh-bénard turbulence," Eur. Phys. J. B 40, 223 (2004).

${ }^{45}$ M. A. Rubio, P. Bigazzi, L. Albavetti, and S. Ciliberto, "Spatiotemporal regimes in rayleigh-bénard convection in a small rectangular cell," J. Fluid Mech. 209, 309-334 (1989).

${ }^{46}$ F. Chillà, S. Ciliberto, C. Innocenti, and E. Pampaloni, "Boundary layer and scaling properties in turbulent thermal convection," Il Nuovo Cimento D 15, 1229 (1993).

${ }^{47}$ S. B. Dalziel, G. O. Hugues, and B. R. Sutherland, "Synthetic schlieren," in 8th International Symposium on flow visualization (1998).

${ }^{48}$ L. Venkatakrishnan and G. E. A. Meier, "Density measurements using the background oriented schlieren technique," Experiments in Fluids 37, 237-247 (2004).

${ }^{49}$ A. Fincham and G. Delerce, "Advanced optimization of correlation imaging velocimetry algorithms," Exp. Fluids Suppl. 29, S13-S22 (2000).

${ }^{50} \mathrm{~S}$. Grossmann and D. Lohse, "Multiple scaling in the ultimate regime of thermal convection," Phys. fluids 23, 045108 (2011).

${ }^{51}$ X. He, D. Funfschilling, H. Nobach, E. Bodenschatz, and G. Ahlers, "Transition to the ultimate state of turbulent rayleighbénard convection," Phys. Rev. Lett. 108, 024502 (2012).

${ }^{52} \mathrm{M}$. Berhanu, G. Verhille, J. Boisson, B. Gallet, C. Gissinger, S. Fauve, N. Mordant, F. Pétrélis, M. Bourgoin, P. Odier, J.F. Pinton, N. Plihon, S. Aumaître, A. Chiffaudel, F. Daviaud, B. Dubrulle, and C. Pirat, "Dynamo regimes and transitions in the vks experiment," Eur. Phys. J. B 77, 459-468 (2010).

${ }^{53}$ B. Saint-Michel, B. Dubrulle, L. Marié, F. Ravelet, and F. Daviaud, "Forcing-type-dependent stability of steady states in turbulent swirling flow," Submitted to Phys. Rev. Lett. (2013), arXiv:1301.1810v2.

${ }^{54}$ L. Landau and E. Lifshitz, Fluid Mechanics, 2nd ed., Course of Theoretical Physics, Vol. 6 (1987). 